\title{
An Energy-efficient Transmission Scheme for Wireless Sensor Network with DSC and virtual MIMO
}

\author{
Guoqiang Zheng ${ }^{1}$, Fangge $\mathrm{Nie}^{1}$, Bing $\mathrm{Li}^{1}{ }^{1}$ Huahong $\mathrm{Ma}^{1}$, Jishun $\mathrm{Li}^{2}$, Yujun Xue ${ }^{2}$, \\ and Peipei $\mathrm{Li}^{1}$ \\ ${ }^{1}$ School of Electronic Information Engineering, Henan University of Science and \\ Technology, Luoyang, 471003, China \\ ${ }^{2}$ Henan Key Laboratory for Machinery Design and Transmission System, Henan \\ University of Science and Technology, Luoyang, 471003, China \\ E-mail :lyzhenggq@126.com
}

\begin{abstract}
Energy efficiency is one of the primary performance parameters in wireless sensor network (WSN). The data collected by neighboring nodes in the high density WSN tend to have a high correlation which is directly transmitted to the sink node (DGN) will seriously reduce the energy efficiency of wireless sensor networks. For improving the energy efficiency of high node density WSN, We introduce distributed source coding (DSC) which can compress the high correlation source data into WSN based on virtual MIMO. In the simulation results indicate that DSC can effectively reduce the energy consumption in data transmission and improve the energy efficiency of the whole WSN.
\end{abstract}

Keywords: Virtual-MIMO, WSNs, DSC, Cooperative Communications

\section{Introduction}

In recent years, wireless sensor network(WSN) has achieved great progress whit more and more new technology being applied to WSN, which improves the suitability and diversity of WSN. However, the limiting energy of WSN nodes is still no improvement and energy saving is still a major problem. Energy consumption in WSN is mainly caused by the communication of the nodes, thus reducing the communication node is the primary means for energy saving. Especially in large-scale WSN, communication energy consumption will increase dramatically with the increasing communication distance. If a certain number of nodes prematurely deplete their energy, they will segment the network into a lot of island, which can seriously affect the reliability of wireless sensor networks. In order to enhance the reliability of WSN, it is necessary to increase the density of nodes. However, the correlation of data collected by adjacent nodes also increases with the increasing density of node. These high-relevant data is transferred directly to the data gather node (DGN) , which seriously reduce the energy efficiency of WSN.

Multiple-Input Multiple-Output (MIMO) technology can exponentially increase the radio channel capacity, the transmission distance and the performance of bit error rate by the transmission of information [1]. But the volume of WSN nodes are generally relatively small, it is difficult to install multiple antenna systems on a single node. Applying directly the MIMO to wireless sensor networks is unpractical. To solve this problem, the researchers combined collaborate communication with MIMO technology and propose virtual MIMO technology. The basic idea of virtual MIMO is that a single antenna node shares antennas with other nodes to implement virtual multi-antenna system, and then communicates with the multi-antenna systems at the distal end. Virtual MIMO allows wireless sensor network to obtain an effect similar to MIMO communication, and reduces the energy consumption of the entire network. Currently, researchers have made a 
lot of good virtual MIMO communication scheme, such as CMIMO [2], HV-MIMO [3], CCP [4], MIMO-CCRN [5] and the like. These virtual MIMO communication scheme solve the problem of energy consumption in WSN communication, but they do not solve low energy efficiency situation in the large-scale high-density WSN. To solve this problem, this article introduces distributed source coding (Distributed Source Coding, DSC) into the WSN based on virtual MIMO. DSC can compress the high correlation source data to improve energy efficiency and extend the network lifetime. Further, since the data is compressed, the data length of the transmission node is reduced, which reduces more energy consumption of communication.

DSC is based on the theorem proved by Slepian and Wolf in 1970s [9]. The fascinating aspect of DSC is that efficient compression of two or more sources can be achieved by separate encoding and joint decoding. Slepian and Wolf give lossless compression theoretical limit of DSC for two related source using information entropy, called S-W Limit. For example, there are two related sources, $X_{1}$ and $X_{2}$, which are separately encoded according to Slepian and Wolf theories with the code rates being respectively $R_{x 1}$ and $R_{\mathrm{X} 2}$. The region of $\mathrm{R}_{\mathrm{x} 1}$ and $\mathrm{R}_{\mathrm{x} 2}$, as shown in Figure 1 , is bounded by the following inequalities:

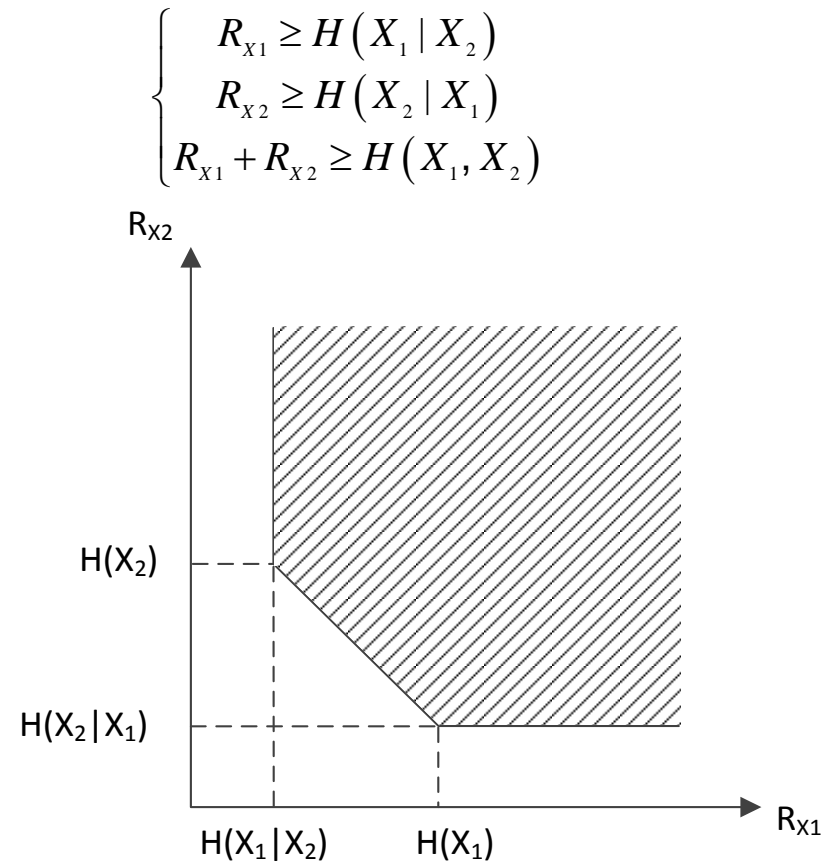

Figure 1. Rate Region for Slepian-Wolf Coding (Two Sources)

Due to the low coding complexity, good rate-distortion and error resilience performance, DSC has been widespread concern of scholars. There has also been a lot of excellent DSC scheme, which can be roughly divided into two categories: asymmetric DSC and symmetric DSC.

For asymmetric DSC scheme, the most representative is distributed source coding using syndromes (DISCUS)[7]. In DISCUS, one source transmits its syndromes to achieve data compression; another source sends its raw data directly as side information. At the receiving end, the decoder recovers the compressed data using the received the side information and the syndromes through jointly decoding. On the basis of DISCUS, researchers have proposed a number of asymmetric DSC scheme using turbo codes and low density parity check (LDPC) [8-10].

For asymmetric DSC scheme, the most representative is the scheme proposed by Sartipi [11]. In Sartipi's scheme, two sources are encoded using LDPC firstly. Then one source sends its corresponding parity bits and only the first half of the information bits, 
the other sends its corresponding parity bits and the second half of the information bits. The decoder recombines the two parts of information bits to form the of noise version of the original information bits, and then using message passing decoding algorithm to recover the original data. Later, Sartipi extends this scheme to achieve a different code rate[12].

\section{Network Model}

We set up a wireless sensor network has the following features:

a) The study object of this paper is the high node density and high scale WSN which uses clustering approach to management and data collected by nodes of the same cluster are high correlation.

b) Similar to the existing virtual MIMO based wireless sensor network, each cluster has a cluster head $(\mathrm{CH})$ to manage the number nodes, and each $\mathrm{CH}$ can select a number of cooperative nodes (CNs) from its neighbor nodes to form a virtual multi-antenna array.

c) Different from ordinary node, DGN's energy is not limited. Moreover DGN can support multi-antenna array and has a strong computing power.

d) For convenience of description, this paper use a virtual MIMO communication link consisting of some adjacent source nodes (SNs), a DGN, a $\mathrm{CH}$ and a $\mathrm{CN}$ to research the effects of DSC to WSN based on virtual MIMO. The communications chain path shown in Figure 2, where the distance between $\mathrm{CH}$ and $\mathrm{CN}$ is far less than the distance of $\mathrm{CH}$ to DGN.

e)

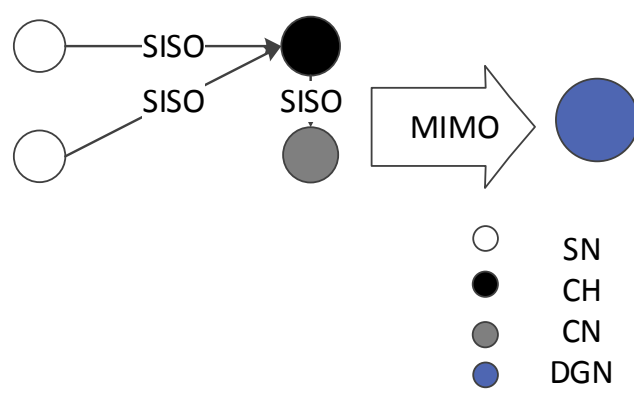

Figure 2. A Virtual MIMO Communication Link

\section{Energy Consumption of WSN based on Virtual MIMO}

The process of virtual MIMO communication is generally divided into two stages: intra-cluster communication phase and inter-cluster communication phase. In the intra-cluster communication phase, the source nodes send the data to the cluster head, and then cluster head forwards the data to cooperative node and complete space-time coding. In inter-cluster communication phase, cluster head and cooperative nodeconstitute a virtual multi-antenna transmission end and transmit the source data to the destination node [1].

Firstly, we must establish energy consumption model of wireless sensor network node firstly. In data transmission, the total power consumption can be divided into two main components [13]: the power consumption of all the power amplifiers $\mathrm{P}_{\mathrm{PA}}$ and the power consumption of all other circuit blocks $\mathrm{P}_{\mathrm{C}}$.

$\mathrm{P}_{\mathrm{PA}}$ can be expressed as:

$$
P_{P A}(d)=(1+\alpha) P_{\text {out }}(d)
$$

Where $\mathrm{d}$ is the distance of nodes; $\alpha$ is a factor related to the drain efficiency; $\mathrm{P}_{\text {out }}(\mathrm{d})$ 
can be calculated using the following formula:

$$
P_{\text {out }}(d)=\bar{E}_{b} R_{b} \frac{(4 \pi)^{2} d^{k} M_{l} N_{f}}{G_{t} G_{r} \lambda^{2}}
$$

Where $\mathrm{k}$ is the path loss; $\mathrm{G}_{\mathrm{t}}$ and $\mathrm{G}_{\mathrm{r}}$ are the antenna gains; $\lambda$ is the wavelength; $\mathrm{M}_{1}$ is the link margin for compensating the hardware process variations and other additive background noise or interference; $\mathrm{N}_{\mathrm{f}}$ is the receiver noise Figure; $\mathrm{R}_{\mathrm{b}}$ is the bit rate; $\bar{E}_{b}$ is the average energy per bit required for a given bit-error-rate (BER), which can be calculated using the following equation:

$$
\begin{aligned}
\bar{P}_{b}= & \frac{1}{2^{N_{T} N_{R}}}\left(1-\frac{1}{\sqrt{1+2 N_{0} / \bar{E}_{b}}}\right)^{N_{T} N_{R}} \times \\
& \sum_{k=0}^{N_{T} N_{R}-1} \frac{1}{2^{k}}\left(\begin{array}{c}
N_{T} N_{R}-1+k \\
k
\end{array}\right)\left(1+\frac{1}{\sqrt{1+2 N_{0} / \bar{E}_{b}}}\right)^{k}
\end{aligned}
$$

Where $\bar{P}_{b}$ is the given BER; $\mathrm{N}_{\mathrm{T}}$ and $\mathrm{N}_{\mathrm{R}}$ are the number of the sending node and the receiving node; $\mathrm{N}_{0}$ is the noise power density.

$\mathrm{P}_{\mathrm{C}}$ can be expressed as:

$$
\begin{aligned}
P_{C} \approx & N_{T}\left(\mathrm{P}_{D A C}+\mathrm{P}_{m i x}+\mathrm{P}_{f i l t}\right)+2 \mathrm{P}_{s y n t h}+ \\
& N_{R}\left(\mathrm{P}_{L N A}+\mathrm{P}_{m i x}+\mathrm{P}_{I F A}+\mathrm{P}_{f i l r}+\mathrm{P}_{A D C}\right)
\end{aligned}
$$

Where $\mathrm{P}_{\mathrm{DAC}}, \mathrm{P}_{\text {mix }}, \mathrm{P}_{\mathrm{LNA}}, \mathrm{P}_{\mathrm{IFA}}, \mathrm{P}_{\text {filt }}, \mathrm{P}_{\text {filr }}, \mathrm{P}_{\mathrm{ADC}}$ and $\mathrm{P}_{\text {synth }}$ are the power consumption values for the digital-to-analog converter (DAC), the mixer, the low-noise amplifier (LNA), the intermediate frequency amplifier (IFA), the active filters at the transmitter side, the active filters at the receiver side, the analog-to-digital converter (ADC), and the frequency synthesizer, respectively.

Finally, the total energy consumption per bit can be expressed as:

$$
E_{p b}(\mathrm{~d})=\frac{P_{P A}(d)+P_{C}}{R_{b}}
$$

The energy consumption of DSC-MIMO focuses on the transmission of source data, which happens in intra-cluster communication phase and inter-cluster communication phase. Next, we use (9) to analyze the energy consumption of the two phases.

\subsection{Intra-cluster Communication Phase}

In the intra-cluster communication phase, nodes use SISO mode to transmit the data. Therefore, the energy consumption for transmitting 1 bit original source data is:

$$
E_{p b}^{S I S O}(d)=\left.\sum_{i=1}^{n_{s}} E_{p b}\left(d_{i}\right)\right|_{N_{T}=N_{R}=1}
$$

Where $d_{i}$ is the distance between the source node $i$ to $\mathrm{CH} ; \mathrm{n}_{\mathrm{s}}$ is the number of the source nodes.

\subsection{Inter-cluster Communication Phase}

In the inter-cluster communication phase, the communication is virtual MIMO mode. In Figure 2 , it is a $2 \times 2$ virtual MIMO link. Therefore, the energy consumption for transmitting 1 bit original source data is: 


$$
E_{p b}^{\text {MIMO }}(d)=\frac{R_{b}^{e f f}}{R_{b}}\left[\left.E_{p b}(d)\right|_{N_{T}=2, N_{R}=2}\right]
$$

Where $R_{b}^{e f f}$ is the effective bit rate, which is expressed as:

$$
R_{b}^{e f f}=\frac{\left(F-p N_{T}\right)}{F} R_{b}
$$

Where $F$ is the block size of Space-Time Coding; $p$ is the training overhead factor.

\section{WSN based on DSC and Virtual MIMO}

DSC has two forms: asymmetric DSC and symmetric DSC. In asymmetric DSC, the data forwarded by different source nodes have various data length, which makes it difficult to complete the space time code (STC) and cause a nonuniform energy consumption. Conversely, symmetric DSC does not have the imperfection of the asymmetric DSC and is more appropriate to WSN. A symmetric DSC, proposed by Sartipi [11], is adopted in this paper.

\subsection{The Virtual MIMO Communication with DSC}

Node $\mathrm{x}$ and $\mathrm{y}$ are two adjacent source nodes from the same cluster. They respectively have data $\mathrm{X}$ and $\mathrm{Y}$ to transmit to $\mathrm{CH}$. Data $\mathrm{X}$ and $\mathrm{Y}$ are both $\mathrm{k}$ bits. The correlations of $\mathrm{X}$ and $\mathrm{Y}$ can be modeled by binary symmetric channel model with the transfer probability $\mathrm{p}$ [12]. $\mathrm{X}$ and $\mathrm{Y}$ can be compressed by using the DSC. Node $\mathrm{x}$ and $\mathrm{y}$ respectively transmit the compressed data to the $\mathrm{CH}$ after encoding data $\mathrm{X}$ and $\mathrm{Y}$. $\mathrm{CH}$ forwards the compressed data to its $\mathrm{CN}$ and completes the space time code. Finally, $\mathrm{CH}$ and $\mathrm{CN}$ transmit the compressed data to the DGN through a virtual MIMO link. The transmission procedure is shown in Figure 3.

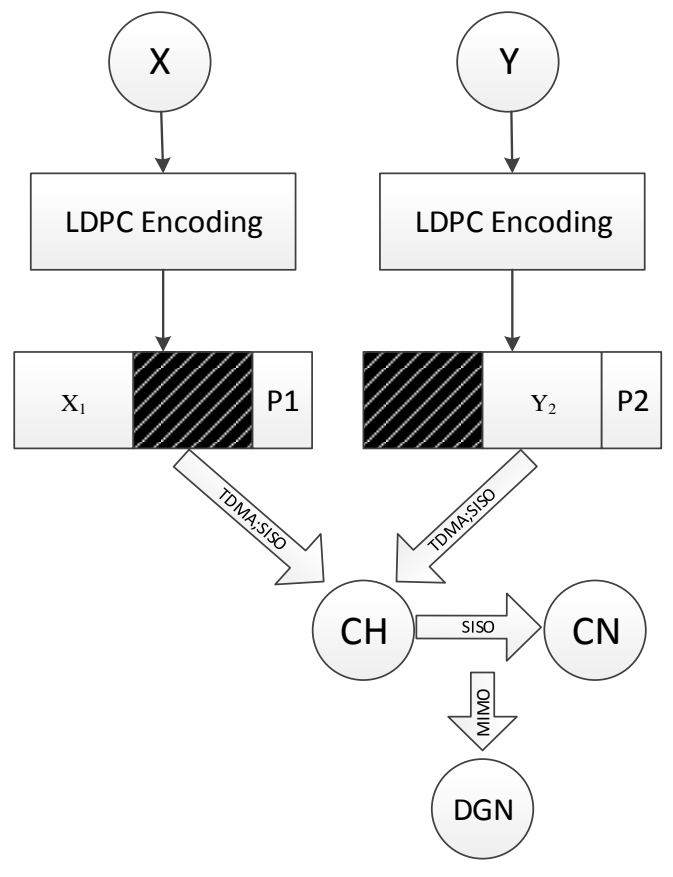

\section{Figure 3. The Transmission Procedure of the Virtual MIMO Communication with DSC}

The encoding process of $\mathrm{X}$ is as follows: $\mathrm{X}$ is fed into a systematic LDPC encoder with a rate $R_{1}$. At the output of the encoder, it sends the corresponding parity bits $P_{X}$ and only the first half of the information bits $\mathrm{X}_{1}$. After the above process, node $\mathrm{x}$ has finished the 
compression of its data X. The compression ratio of DSC is $\mathrm{R}_{\mathrm{x}}(0<\mathrm{Rx}<1)$ which can be calculated by the following formula.

$$
R_{X}=\frac{k / 2+P_{X}}{k}
$$

The code rate of LDPC is $R_{1}$. The relationship between $R_{x}$ and $R_{1}$ can be described by the following formula.

$$
R_{1}=\frac{k}{n}=\frac{1}{R_{X}+1 / 2}
$$

The encoding process of $\mathrm{Y}$ is similar to $\mathrm{X}$ with the modification that for the second source we send the second half of the information bits $\mathrm{Y}_{2}$ along with the corresponding parity bits. The compression ratio of $Y$ is $R_{y}$ which is equal to $R_{x}$. The LDPC code rate of $\mathrm{Y}$ is $\mathrm{R}_{2}$ which is equal to $\mathrm{R}_{1}$. Since the compression rates of both sources are the same, the rates of the corresponding systematic LDPC codes are identical. Therefore, we only need to design a single LDPC code for compressing two correlated sources at the symmetric rate with DSC.

In order to obtain the original data, DGN need to decode the received data. In the decoding process, DGN regroups the data as $\left(\mathrm{X}_{1}, \mathrm{Y}_{2}, \mathrm{P}_{\mathrm{X}}\right)$ and $\left(\mathrm{X}_{1}, \mathrm{Y}_{2}, \mathrm{P}_{\mathrm{Y}}\right) .\left(\mathrm{X}_{1}, \mathrm{Y}_{2}, \mathrm{P}_{\mathrm{X}}\right)$ can be seen as $\left(\mathrm{X}+\mathrm{n}, \mathrm{P}_{\mathrm{X}}\right)$, where $\mathrm{n}$ is the noise. $\left(\mathrm{X}_{1}, \mathrm{Y}_{2}, \mathrm{P}_{\mathrm{Y}}\right)$ can be seen as $\left(\mathrm{Y}+\mathrm{n}, \mathrm{P}_{\mathrm{Y}}\right)$, where $\mathrm{n}$ is the noise. DGN decodes $\left(\mathrm{X}_{1}, \mathrm{Y}_{2}, \mathrm{P}_{\mathrm{X}}\right)$ and $\left(\mathrm{X}_{1}, \mathrm{Y}_{2}, \mathrm{P}_{\mathrm{Y}}\right)$ through posterior probability $(\mathrm{BP})$ decoding algorithm and recover the original data $\mathrm{X}$ and $\mathrm{Y}$.

\subsection{Analysis of Energy Consumption}

In the intra-cluster communication phase, node $\mathrm{x}$ and $\mathrm{y}$ respectively send compressed data to the cluster head node, and then the cluster head node forwards the receive data to the cooperating nodes. During this process, the energy consumption of the WSN can be calculated as followed.

$$
E_{D S C}^{I N T R A}=k R_{x} E_{p b}^{S I S O}\left(d_{x}\right)+k R_{y} E_{p b}^{S I S O}\left(d_{y}\right)+\left(k R_{x}+k R_{y}\right) E_{p b}^{S I S O}\left(d_{C N}\right)
$$

Where $\mathrm{k}$ is the data length of node $\mathrm{x}$ and $\mathrm{y} . \mathrm{R}_{\mathrm{x}}$ and $\mathrm{R}_{\mathrm{y}}$ are the compressing rate of node $\mathrm{x}$ and $\mathrm{y}$, moreover, $\mathrm{R}_{\mathrm{x}}=\mathrm{R}_{\mathrm{y}} . \mathrm{d}_{\mathrm{x}}$ is the distance between node $\mathrm{x}$ and $\mathrm{CH} . \mathrm{d}_{\mathrm{y}}$ is the distance between node $\mathrm{y}$ and $\mathrm{CH}$. $\mathrm{d}_{\mathrm{CN}}$ is the distance between $\mathrm{CN}$ and $\mathrm{CH}$.

In the intra-cluster communication phase, $\mathrm{CH}$ and $\mathrm{CN}$ form a virtual multi-antenna array and transmit the data to the DGN through a virtual MIMO link. During this process, the energy consumption of the WSN can be calculated as followed.

$$
E_{D S C}^{I N T E R}=2 k R_{x} \times E_{p b}^{M I M O}\left(d_{D G N}\right)
$$

Where $\mathrm{d}_{\mathrm{DGN}}$ is the distance between $\mathrm{CH}$ and DGN.

\section{Simulation Analyses}

To verify the energy conservation effect of DSC, we establish a simulation platform to compare the energy consumption in different communication phase WSN based on virtual MIMO and DSC. The main simulation parameters are shown in Table 1, which is same with [13].

Table 1. Simulation Parameters

\begin{tabular}{c|c}
\hline$\lambda=0.125 \mathrm{~m}$ & $\alpha=0.47$ \\
$\mathrm{G}_{\mathrm{t}} \mathrm{G}_{\mathrm{r}}=5 \mathrm{dBi}$ & $\mathrm{N}_{0}=-174 \mathrm{dBm} / \mathrm{Hz}$ \\
$\mathrm{B}=10 \mathrm{KHz}$ & $\mathrm{P}_{\text {mix }}=30.3 \mathrm{~mW}$ \\
$\mathrm{P}_{\mathrm{IFA}}=20 \mathrm{~mW}$ & $\mathrm{P}_{\text {syn }}=50 \mathrm{~mW}$ \\
$\mathrm{P}_{\text {filt }}=\mathrm{P}_{\text {filr }}=2.5 \mathrm{~mW}$ & $\mathrm{P}_{\mathrm{LNA}}=20 \mathrm{~mW}$ \\
$\mathrm{~N}_{\mathrm{f}}=10 \mathrm{~dB}$ & $\mathrm{M}_{\mathrm{l}}=40 \mathrm{~dB}$ \\
\hline
\end{tabular}


Figure 4 shows the energy consumption of the WSN based on DSC and virtual MIMO and the WSN based on virtual MIMO in the intra-cluster communication phase. In the Figure, the energy consumption performance of the WSN based on DSC and virtual MIMO is better than WSN based virtual MIMO. The introduction of DSC can help the WSN saving about $25 \%$ energy in the intra-cluster communication phase. DSC can compress the related source data and reduce the transmission data. Therefore, the WSN based on DSC is more energy-saving in intra-cluster communication, and the performance is growing with the increasing number of source nodes. In the intra-cluster communication phase, WSN nodes in use SISO way communication.

Figure 5 shows the energy consumption of the WSN based on DSC and virtual MIMO and the WSN based on virtual MIMO in the intra-cluster communication phase. Similar to Figure 4, the WSN based on DSC and virtual MIMO has a better energy consumption performance than the WSN based on virtual MIMO. However, the improvement is only $14 \%$. This is due to the increased bit error rate at DGN for decoding the compressed data. To compensate for this loss of performance, the $\mathrm{CH}$ and $\mathrm{CN}$ need to increase the transmission power. In the intra-cluster communication phase, $\mathrm{CH}$ is not required to decode these compressed data, thus the increasing bit errorcaused by DSC does not affect the energy consumption performance. However, the increasing power does not exceed energy savings brought by DSC. Therefore the use of DSC and virtual MIMO for WSN is more energy efficient in inter-cluster communication phase.

Figure 6 shows the energy efficiency in different transmission mode. We define the energy efficiency as the total energy consumption for transmitting 1 bit original source data. SISO is the transmission mode of intra-cluster communication and MIMO is the transmission mode of inter-cluster communication. In the short distance communication, SISO is more energy efficient than virtual MIMO with DSC and virtual MIMO. This is mainly because that energy consumption from the circuit of nodes is the main part of the whole energy consumption. Virtual MIMO requires two nodes transmitting data simultaneously, so the energy consumption are more. With the increase of the communication distance, the energy consumption from circuit of nodes is substantially constant, while the energy consumption of the amplifier is increasing apace and gradually become the main energy consumption. The advantages of virtual MIMO on energy efficiency have gradually realized. So SISO is more suitable for close communication and virtual MIMO is more suitable for long distance communications. Virtual MIMO with DSC can reduce the length of the data sent by the nodes, thus further enhancing energy efficiency.

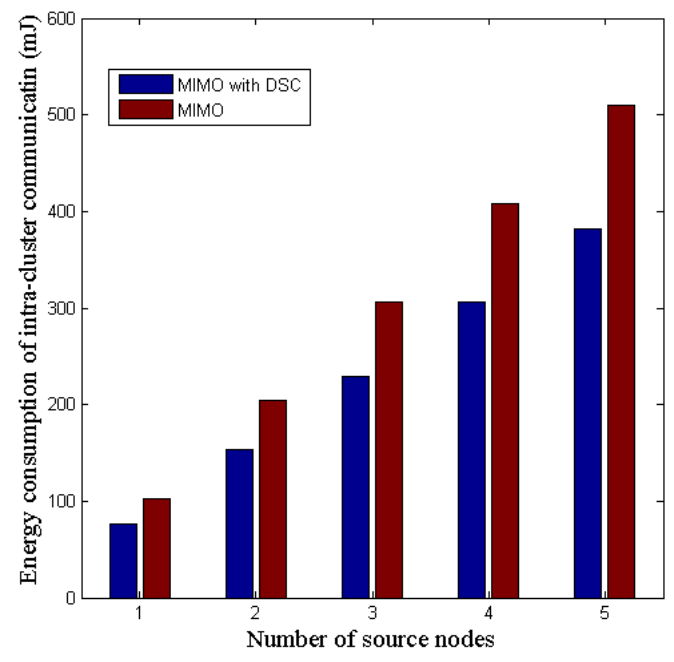

Figure 4. The Energy Consumption of the WSN based on DSC and Virtual MIMO and The WSN based on Virtual MIMO in the Intra-cluster Communication Phase 


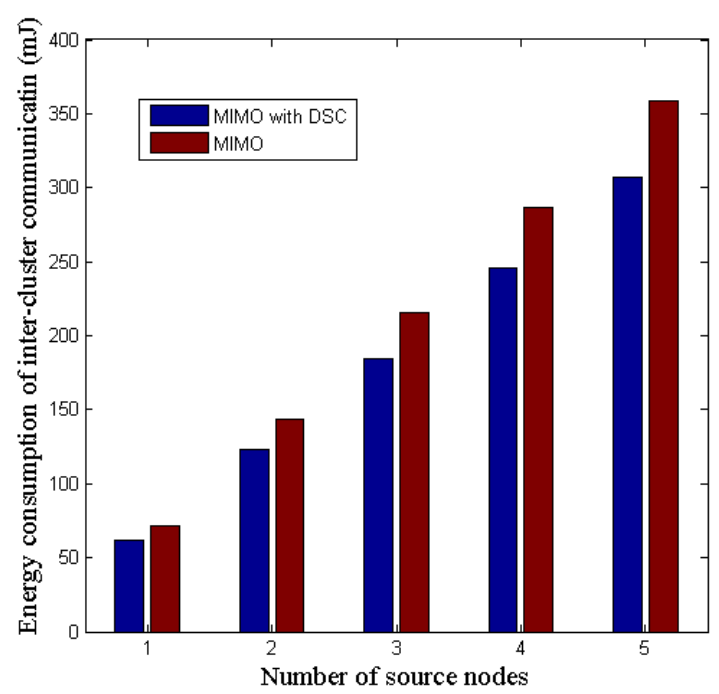

Figure 5. The Energy Consumption of the WSN based on DSC and Virtual MIMO and the WSN based on Virtual MIMO in the Inter-cluster Communication Phase

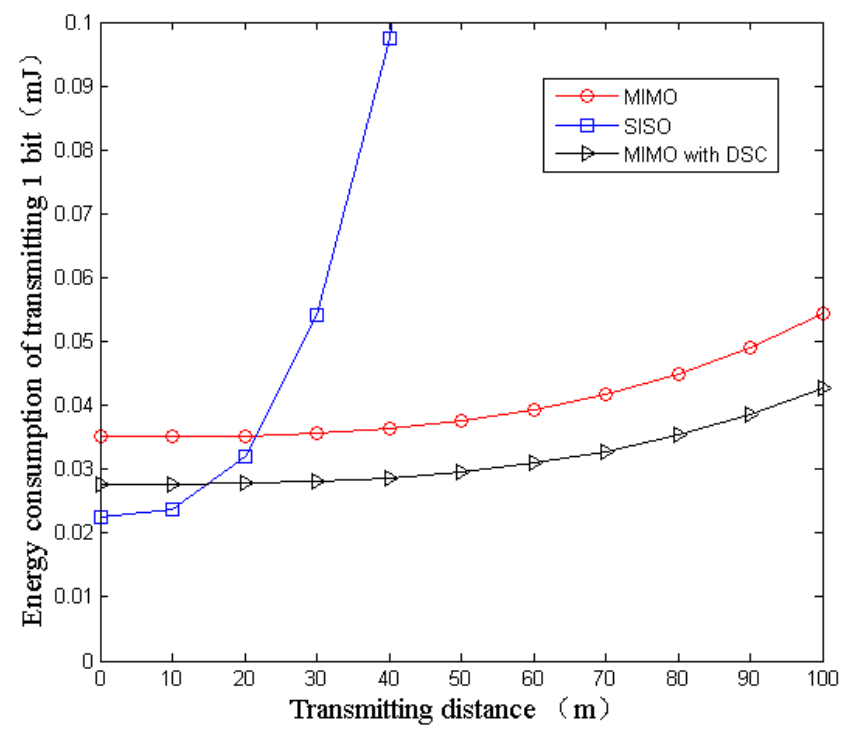

Figure 6 The Energy Consumption in Different Transmission Mode

\section{Conclusion}

In the WSN with high node density, the collected data of neighboring nodes tend to have high correlation. Transmitting these high correlation data directly will waste a lot of energy and reduce energy efficiency of WSN. In this paper, we introduce DSC into the WSN based on virtual MIMO. The DSC can compress the high correlation data and reduce the length of the data that are forward by nodes, which can reduce the energy consumption of communication.

The simulation results show that compared to the WSN based virtual MIMO, the WSN based on DSC and virtual MIMO need less energy during communication and has a better energy efficiency performance. 


\section{Acknowledgment}

This work is supported by the National Basic Research Program of China (No. 2014CB049401), the Project of Basic and Advanced Technology Research of Henan Province of China (No. 132300410001), and the Program for Innovative Research Team (in Science and Technology) in University of Henan Province (No. 15IRTSTHN008).

\section{References}

[1] D. N. Nguyen and M. Krunz ,"Cooperative MIMO in wireless networks: Recent developments and challenges". IEEE Network, vol. 27, (2013), pp. 48-54.

[2] M. Krunz, Z. S. Mohammad and D. N. Nguyen, "Clustering and power management for virtual MIMO communications in wireless sensor networks". AD HOC Networks, vol. 11, (2013), pp. 1571-1587.

[3] M. Nasim, S. Qaisar and S. Lee, "An Energy Efficient Cooperative Hierarchical MIMO Clustering Scheme for Wireless Sensor Networks", Sensors, vol. 12, (2012), pp. 92-114.

[4] D. N. Nguyen and M. Krunz, "A Cooperative Clustering Protocol for Energy Constrained Networks", Proceedings 8th IEEE ComSoc Conf. Sensor, Mesh and Ad Hoc Commun. and Networks, (2011), pp. 574-582.

[5] S. Hua, H. Liu, M. Wu and S. Shivendra, "Exploiting MIMO Antennas in Cooperative Cognitive Radio Networks", Proceedings IEEE INFOCOM, (2011), pp. 2714 - 2722.

[6] D. Slepian and J. K. Wolf, "Noiseless coding of correlated information sources", IEEE trans on Information theory, vol. 19, (1973), pp. 471-480.

[7] S. S. Pradhan and K. Ramchandran, "Distributed source coding using syndromes (DISCUS): design and construction”, Proceedings IEEE Data Compression Conference, (1999), pp. 158-167.

[8] A. Aaron and B. Girod, "Compression with side information using turbo codes", Proceedings IEEE Data Compression Conference, April, (2002), pp. 252-261.

[9] D. Varodayan and B. Girod, "Side-information-adaptive distributed source coding", Image Processing (ICIP), 2010 17th IEEE International Conference on, Sept, (2010), pp. 3729-3732.

[10] A. D. Liveris, Z. Xiong and C. N. Georghiades, "A distributed source coding technique for correlated image using turbo codes", IEEE Comm.Letters, vol. 6, (2002), pp. 379 - 381.

[11] M. Sartipi and F. Fekri, "Distributed source coding in wireless sensor networks using LDPC coding: the entire Slepian-Wolf rate region". Wireless Communications and Networking Conference, 2005 IEEE, (2005) , pp. 1939 - 1944.

[12] M. Sartipi and F. Fekri, "Distributed source coding using short to moderate length rate-compatible LDPC codes: the entire Slepian-Wolf rate region", Communications, IEEE Transactions on, vol. 56, (2008), pp. 400-411.

[13] S. Cui, A. J. Goldsmith and A. Bahai "Energy-efficiency of MIMO and cooperative MIMO techniques in sensor networks". IEEE Journal on Selected Areas in Communications, (2004), pp. 1089-1098.

[14] S. K. Jayaweera, "Virtual MIMO-based cooperative communication for energy-constrained wireless sensor networks",IEEE Transactions on Wireless Communications, vol. 5, (2006), pp. 984-989.

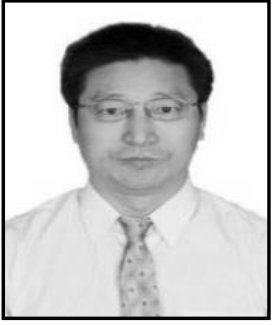

Guoqiang Zheng, he received the Ph.D. degree in Communication and information systems professional from Xi'an Jiaotong University, China, 2008. He is a professor at Henan University of Science and Technology on College of Electronic Information Engineering, China. His research interests include wireless communication technology, network communication protocol and software radio theory.

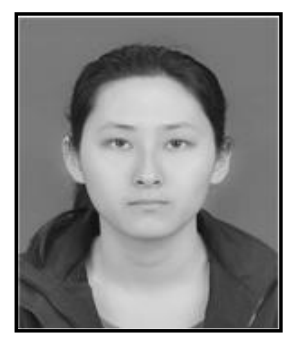

Fangge Nie, she received the B.S. degree in Henan University of Science and Technology, Luoyang, China in 2013. She is currently working towards M.S. degree in Henan University of Science and Technology, Luoyang, China. Her research interests include WSNs and Opportunistic Routing. 

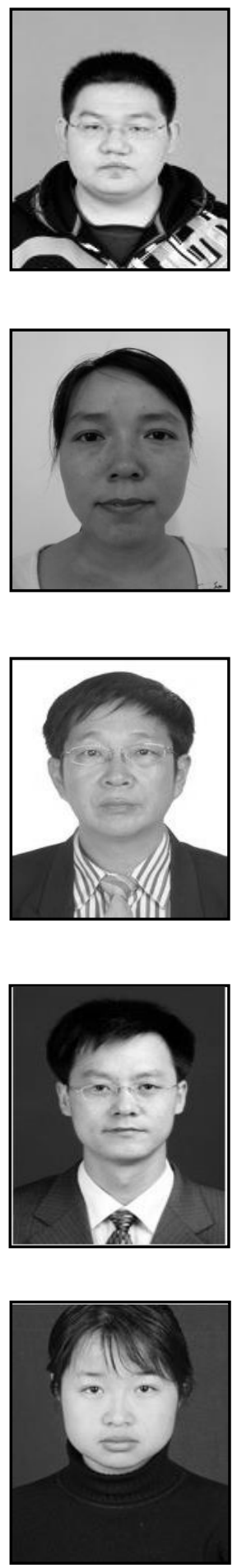

Bing $\mathbf{L i}$, he received the B.S. degree in information engineering in Henan University of Science and Technology, Luoyang, China in 2011. He is currently working towards M.S. degree in Henan University of Science and Technology, Luoyang, China. His research interests include WSNs, virtual MIMO and distributed source coding.

Huahong Ma, she received her master degree in Signal and Information Processing in July 2005 at Yunnan University, China. Now, she is a Ph.D. candidate in Control Science and Engineering at Henan University of Science and Technology. Her main research interests are Crowd Sensing Network and Internet of Things.

Jishun Li, he received the Ph.D. degree in Mechanical Manufacture and Automation from Shanghai Jiaotong University, China, 1996. He is a professor at Henan University of Science and Technology on College of Mechatronics Engineering, China.

Yujun Xue, he received the Ph.D. degree in mechanical design and theory from Shanghai Jiaotong University, China, 2002. He is a professor at Henan University of Science and Technology on College of Mechatronics Engineering, China.

Peipei Li, she received the M.S. degree in Communication and Information System in Nanjing University of Science and Technology, Nanjing, China in 2006. She is a lecturer at Henan University of Science and Technology on College of Electronic Information Engineering, China. 